\section{EREM 76/1}

Journal of Environmental Research Engineering and Management

Vol. 76 / No.1 / 2020

pp. 44-57

DOI 10.5755/j01.erem.76.1.23542
Changes in Plants and Invertebrate Animals Communities along Gradients of Petroleum Products Pollution

\title{
Changes in Plants and Invertebrate Animals Communities along Gradients of Petroleum Products Pollution
}

\section{Djaffer Dib*}

PSEMRVC Laboratory, Department of Biology, Faculty of Biological Sciences and Agronomical Sciences, Mouloud Mammeri University, Tizi-Ouzou, 15000, Algeria

\section{Djamila Sadoudi Ali Ahmed}

PSEMRVC Laboratory, Department of Biology, Faculty of Biological Sciences and Agronomical Sciences, Mouloud Mammeri University, Tizi-Ouzou, 15000, Algeria

\section{*Corresponding author: djaffer.dib@ummto.dz}

In this work, we investigated the effects of petroleum product contamination, caused by petrol station wastes, on the vegetation and soil invertebrate animal communities. Three sites situated near two petrol stations were selected, and the vegetation was inventoried during each season of the year. The invertebrates were sampled in the third site using pitfall traps. Results revealed that petrol station wastes negatively affected soil properties (TPH, N, C, pH and moisture). Similarly, plant species and invertebrate communities were influenced in terms of the abundance, species richness, biological diversity $\left(\mathrm{H}^{\prime}\right)$ and species evenness $(\mathrm{J})$, especially in the most contaminated zones. Some weed plant species showed tolerance to the contamination, as Xanthium strumarium, Avena sp, Conium maculatum and Oxalis pes-caprae, and their establishment may have been facilitated by the disturbances caused by the petrol station wastes. Also, some invertebrate orders, as Isopoda and Panpulmonata, were mainly present in the contaminated zones. The high levels of petroleum product contamination and the changes they may cause in soil properties can affect the vegetation and soil invertebrates by influencing the abundance and species richness and by facilitating the establishment of some species, like weed species in the vegetation case.

Keywords: Vegetation, invertebrates, petroleum products, contamination, petrol station. 


\section{Introduction}

Petroleum products are mixtures composed mainly of hydrocarbons which can cause soil contamination (Henry, 1998; Pinedo, 2013). Some of them are persistent, whilst others are significantly reduced through time by means of many processes (Rhbal et al., 2010), including biodegradation, which depends on many factors (Solano-Serena et al., 2001). Hydrocarbons can cause toxic effects on plant species and soil living organisms when they reach a certain level of concentration (Tang et al., 2011). Hydrocarbon contamination can either positively or negatively affect different environment components, like soil properties $(\mathrm{pH}$, total nitrogen and carbon for example) (Adam and Duncan, 2002; Akpan and Udoh, 2013), germination and growth of plant species (Luhach and Chaudhry, 2012; Fatokun et al., 2015) and macrofauna diversity and abundance (Erstfeld and Snow-Ashbrook, 1999). Transects have already been used to study the effects of pollution gradients on vegetation communities (Dazy et al., 2009) and macrofauna communities (Erstfeld and Snow-Ashbrook, 1999; Grzes', 2009). Ecological analyses can be used to understand changes in vegetation communities (Dazy et al., 2009) and macrofauna communities (Grzes', 2009) along pollution gradients in contaminated sites.

Petroleum products are an indispensable energy source for most countries in the actual context, and their use represents a source of many environmental contaminations and problems. Thus, the biotechnologies are proposed as alternative green solutions for the restoration of polluted sites (Kathl and Khan, 2011; Mandal et al., 2014). Vegetation sampling through pollution gradients can highlight some petroleum products tolerant plant species, which can be proposed for further research in order to determine their potential for the remediation of contaminated sites.

Petrol stations are potential sources of petroleum product soil contaminations. Even if they are subject to several environmental regulations, there are still significant soil contaminations in the environs of petrol stations, due mainly to fuel spills and fuel tanks leaks (Borowiec et al., 2008; Moschini et al., 2005; Parvizi Mosaed et al., 2015). We know that fuel spills from petrol stations can affect the surrounding environment and people's health (Alharbi et al., 2018; Pearson and Oudijk, 1993); consequently, an investigation about the effects of those petrol station wastes on their surrounding environment will provide some interesting information about the changes they may cause in the soil and the response of living organisms to this situation. In this study, we selected three sites near two petrol stations, and we investigated the effects of the petroleum product contamination on the vegetation and the invertebrate communities, and tried to identify some potential tolerant species to such contaminations.

\section{Materials and Methods}

\section{Sites description}

This work was performed near two petrol stations, in Freha $\left(36^{\circ} 44,10^{\prime} 40^{\prime \prime} \mathrm{N}, 4^{\circ} 18,24^{\prime} 83^{\prime \prime} \mathrm{E}\right)$ and Idjeur $\left(36^{\circ} 39^{\prime} 30.58^{\prime \prime} \mathrm{N}, 4^{\circ} 27^{\prime} 54.51{ }^{\prime \prime} \mathrm{E}\right)$, two regions located in the middle-north of Algeria. The climate is Mediterranean continental with cold winters and hot dry summers. Altitudes in Freha and Idjeur are $124 \mathrm{~m}$ and $470 \mathrm{~m}$, respectively. Three study sites were selected: the first site is located near the Idjeur petrol station and two other sites near the Freha petrol station. The study sites were

subject to liquid discharges, contaminated with petroleum products emanating from the petrol stations. The wastes are mainly composed of fuels (petrol and diesel), waste oils, lubricating oils and water.

\section{Sampling design}

Based on the vegetation structure, it was possible to identify four vegetation gradients that were supposed to be related to the contamination. Then, two transects 
(T1 and T2) were established from the rejection point (low vegetation cover) to a distant area supposed to be unpolluted (important vegetation cover), and four zones were delimited in each transect. The first zone is located near the rejection point and the fourth zone is located in the unpolluted zone. This sampling design was used for soil, vegetation and invertebrates sampling (Figure 1 and 2).

Fig. 1. Sampling site 1 (Idjeur petrol station).

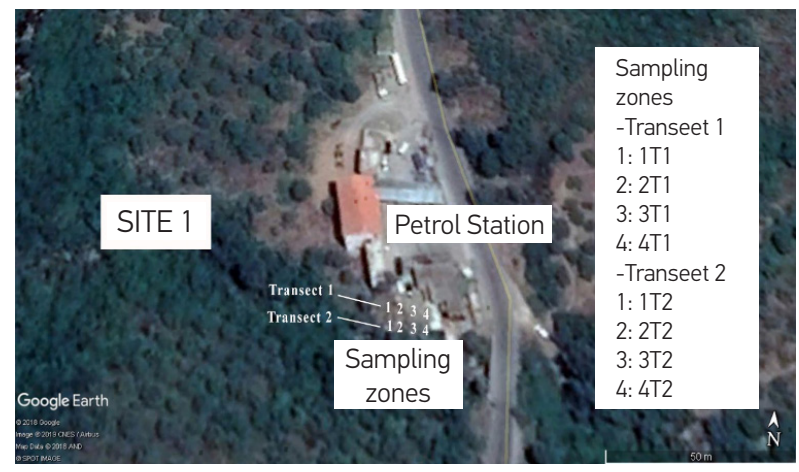

Fig. 2. Sampling site 1 (Idjeur petrol station).

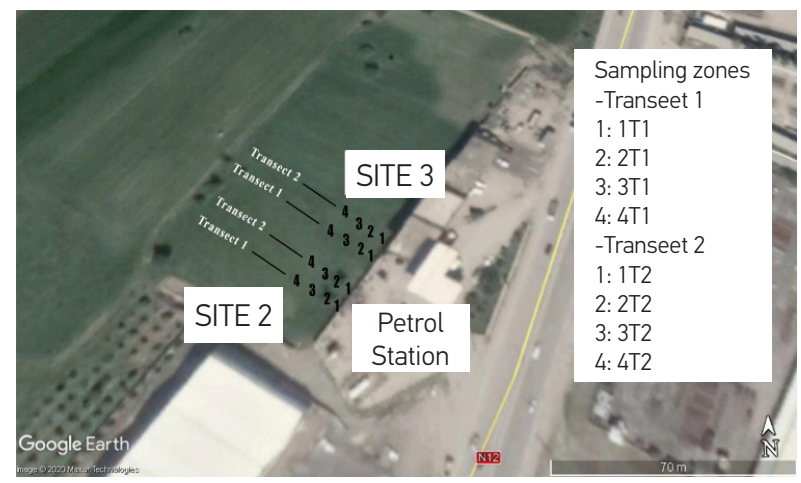

Soil sampling was performed in May 2018. For each zone, soil samples were collected at a depth of $20 \mathrm{~cm}$, put in plastic bags and conserved at $4^{\circ} \mathrm{C}$ before being analysed. Soil physical-chemical analyses concerned the granulometric composition, $\mathrm{pH}$, electrical conductivity (EC), total Kjeldahl nitrogen (N), carbon (C) and moisture. These analyses were performed at the National Institute of Forester Research (INRF) based in Algiers. Total petroleum hydrocarbons (TPH) analyses were performed at the laboratory of petrochemical synthesis, in the Faculty of Hydrocarbons and Chemistry (Boumerdes University), using the Mid-IR laser spectroscopy method. TPH analyses were only performed for site 3. Macherey Nagel qualitative oil test paper was used to determine the polluted and the unpolluted zones through the transects of sites 1 and 2 .

\section{Vegetation sampling}

Four surveys were performed (in November 2017, January 2018, March 2018 and May 2018). For each zone, we delimited a quadrat of $50 \times 50 \mathrm{~cm}$, in which we performed the vegetation inventory and the estimation of plant species abundance-dominance developed by Braun-Blanquet et al. (1932). Species richness (S) and abundance (the number of individuals, noted as N), in each zone were estimated. Shannon-Weaver's diversity $\left(H^{\prime}\right)$, Pielou's evenness $(J)$ and Sorensen-Dice coefficient (DSC) were calculated according to Shannon and Weaver (1964), Pielou (1966), Sorensen (1948) and Dice (1945), respectively.

\section{Invertebrates sampling}

Soil invertebrates sampling was performed in the third site using pitfall trapping during ten days in August 2018. We used plastic pitfall traps of $9 \mathrm{~cm}$ in diameter and $17 \mathrm{~cm}$ in height. In each zone of the two transects, one pitfall trap was placed and was third party filled with water, saturated with salt, and a small amount of liquid detergent was added. Pitfall traps were checked every two days (five collections), and trapped individuals were placed in plastic bottles containing $70^{\circ}$ alcohol. Invertebrates were identified until the order, and the approximate number of species was determined without the species being identified. Order abundance, number of orders and species richness (S) were estimated for each zone. Shannon-Weaver's diversity $\left(H^{\prime}\right)$, Pielou's evenness $(J)$ and Sorensen-Dice coefficient (DSC) were calculated.

\section{Data analyses}

ANOVA followed by the Newman-Keuls test was performed to highlight the significant differences $(p<0.05)$. The Pearson linear correlation method was used to investigate the relationships between soil properties and ecological indexes. All tests were performed under Statistica 7.1 software (@StatSoft). 


\section{Results and Discussion}

Soil analyses results are represented in Table 1. TPH analyses on site 3 showed a gradual decrease in the contamination through the different zones, from zones 1 (most polluted zones, with $336.35 \mathrm{mg} / \mathrm{kg}$ and 333.62 $\mathrm{mg} / \mathrm{kg}$ ) to zones 4 (no pollution detected). Qualitative tests indicated the presence of contaminated zones in sites 1 and 2, and carbon rates in these two sites suggest a clear differentiation between zones 1 and the other zones. For each site, the granulometric composition was fairly similar across the different zones. However, we observed some variations, in particular for sand rates on site 3. Carbon, TPH and moisture rates decreased progressively from zones 1 to zones 4 while total nitrogen showed an opposite tendency. No particular tendency was found for the electrical conductivity. In site 3, the Pearson linear correlation showed a very strong positive correlation $(p<0.05)$ between TPH and $\mathrm{C} / \mathrm{N}$ ratio (Table 2 ). The hydrocarbon contamination caused an increase in carbon rates and moisture in all sites, and potentially a decrease in $\mathrm{pH}$ values for sites 1 and 2. Similarly, Akpan and Udoh (2013) reported an acidification of the soil and an increase in carbon rates after a contamination by diesel oil. The petrol stations wastes are composed partly of water, which explains the increase in moisture rates. The $\mathrm{C} / \mathrm{N}$ ratio was altered by the contamination, resulting in an increase of $\mathrm{C} / \mathrm{N}$ ratios in zones 1 and a decrease when moving to zones 4 . It was reported by Adam and Duncan (2003) that changes in the $\mathrm{C} / \mathrm{N}$ ratio may negatively affect the vegetation development. Parvizi Mosaed et al. (2015) have reported high levels of TPH in the soils of many petrol stations and have observed some effects on physical-chemical properties of the soils. The homogeneous groups (indicated in Table 1 by different letters) highlight the significant differences through the different zones. Zones 1 (most contaminated zones) are often represented in a separate group, in opposition to the other 3 zones, suggesting an important influence of the petrol stations wastes on soil properties in zones 1.

The total species abundances in each zone presented a general significant tendency $(p<0.05)$, showing a progressive increase from zones 1 to zones 4 (Table
3). The unpolluted zones presented the biggest abundances. This tendency is not systematically observed when we look at the species richness, but we can see that zones 1 always present the lowest species richness (Table 3 ). These observations suggest a negative influence caused by the contamination and the alteration of soil properties. The homogeneous groups (indicated in Table 3 by different letters) highlight the significant differences through the different zones. Zones 1 are often represented in a separate group, in opposition to the other 3 zones, suggesting that the high contamination levels in zone 1 have an important influence on its vegetation.

Abundance-dominance of plant species is represented in Tables 4, 5 and 6. Three species present a good distribution through the different zones in all sites, establishing even in zones 1. Those species are Avena sp, Conium maculatum and Oxalis pes-caprae. However, there are some species that present a good distribution, but they are station specific, as Elymus repens in site 3, Vicia sativa, Cerinthe major and Senecio vulgaris in site 1, and Galactites tomentosa in site 2. Xanthium strumarium showed a preference for zones 1 , especially in sites 2 and 3 , presenting a tendency to dominate the zones 1; thus, it is rare in zones 3 and absent in zones 4 . There are some plant species that avoided the polluted zones, as Beta vulgaris, Senecio vulgaris, Malva sylvestris, Sonchus oleraceus, Galactites tomentosa and Scolymus hispanicus.

Many studies showed the negative effects of hydrocarbon contamination on the growth and germination of plant species, as Fatokun et al. (2015), Adam and Duncan (2002) and Luhach and Chaudhry (2012). Also, these studies revealed that the effects depend on the plant species because not all the species react in the same way to contamination. Likewise, our observations reveal that the contamination affected the species richness, especially in zones 1 , the poorest zones in term of species. Some plant species succeeded to establish in the polluted zones, as Xanthium strumarium, Avena sp, Conium maculatum and Oxalis pescaprae. Nevertheless, other species as Beta vulgaris, Senecio vulgaris, Malva sylvestris, Sonchus oleraceus, 
Table 1. Soil physical-chemical properties in each transect (T1 and T2) of the three sites sampled

\begin{tabular}{|c|c|c|c|c|c|c|c|c|c|c|c|}
\hline Sites & Zones & $\mathrm{pH}$ & Clay & Silt & Sand & $\mathrm{EC}$ & c & N & $\mathrm{C} / \mathrm{N}$ & Moisture & TPH \\
\hline \multirow{8}{*}{1} & $1 \mathrm{~T} 1$ & $7.6^{\mathrm{a}}$ & 28.35 & $34.82^{\mathrm{a}}$ & 36.82 & 0.42 & $1.03^{a}$ & 0.03 & $34.50^{\mathrm{a}}$ & $24.43^{\mathrm{a}}$ & Polluted \\
\hline & $1 \mathrm{~T} 2$ & $7.9^{a}$ & 29.34 & $35.45^{\mathrm{a}}$ & 35.21 & 0.4 & $1.04^{a}$ & 0.03 & $34.67^{a}$ & $24.53^{a}$ & Polluted \\
\hline & $2 \mathrm{~T} 1$ & $8.2^{b}$ & 36.7 & $36.33^{a}$ & 26.97 & 0.34 & $0.23^{b}$ & 0.05 & $4.53^{b}$ & $24.35^{\mathrm{a}}$ & Polluted \\
\hline & $2 \mathrm{~T} 2$ & $8.2^{b}$ & 41.8 & $40.18^{\mathrm{a}}$ & 18.02 & 0.43 & $0.15^{b}$ & 0.04 & $3.78^{b}$ & $24.36^{a}$ & Polluted \\
\hline & $3 T 1$ & $8.4^{b}$ & 39 & $34.27^{a}$ & 26.73 & 0.38 & $0.46^{c}$ & 0.05 & $9.19 \mathrm{c}$ & $20.93^{b}$ & Polluted \\
\hline & $3 \mathrm{~T} 2$ & $8.4^{b}$ & 33.45 & $35.58^{\mathrm{a}}$ & 30.97 & 0.34 & $0.77^{c}$ & 0.1 & $7.67^{c}$ & $20.48^{b}$ & Polluted \\
\hline & $4 \mathrm{~T} 1$ & $8.2^{b}$ & 38.5 & $23.7^{b}$ & 37.81 & 0.4 & $0.15^{b}$ & 0.14 & $1.08^{d}$ & $17.10^{c}$ & Unpolluted \\
\hline & $4 \mathrm{~T} 2$ & $8.2^{b}$ & 22.4 & $26.8^{b}$ & 52.4 & 0.32 & $0.08^{b}$ & 0.1 & $0.76^{d}$ & $17.48^{c}$ & Unpolluted \\
\hline \multirow{8}{*}{2} & $1 \mathrm{~T} 1$ & 7.5 & 5.05 & $43.8^{a}$ & 51.15 & 0.22 & $0.95^{\mathrm{a}}$ & $0.04^{a}$ & $23.75^{\mathrm{a}}$ & $21.34^{a}$ & Polluted \\
\hline & $1 \mathrm{~T} 2$ & 7.4 & 3.55 & $43.9^{a}$ & 52.55 & 0.18 & $0.92^{\mathrm{a}}$ & $0.05^{\mathrm{a}}$ & $18.37^{a}$ & $21.08^{a}$ & Polluted \\
\hline & $2 \mathrm{~T} 1$ & 7.4 & 4.1 & $31.5^{b}$ & 64.39 & 0.54 & $0.38^{b}$ & $0.05^{\mathrm{a}}$ & $7.67^{b}$ & $17.25^{b}$ & Polluted \\
\hline & $2 \mathrm{~T} 2$ & 8.5 & 8.45 & $30.4^{b}$ & 61.15 & 0.63 & $0.46^{b}$ & $0.1^{a}$ & $4.59^{b}$ & $17.33^{b}$ & Polluted \\
\hline & $3 \mathrm{~T} 1$ & 8.1 & 5.65 & $31.48^{b}$ & 62.87 & 0.16 & $0.77^{c}$ & $0.1^{\mathrm{a}}$ & $7.67^{b}$ & $17.16^{b}$ & Polluted \\
\hline & $3 \mathrm{~T} 2$ & 7.9 & 4.2 & $31.52^{b}$ & 64.27 & 0.88 & $0.69^{c}$ & $0.14^{a}$ & $4.94^{b}$ & $17.03^{b}$ & Polluted \\
\hline & $4 \mathrm{~T} 1$ & 8 & 9.5 & $28.64^{b}$ & 61.86 & 0.88 & $0.61^{c}$ & $0.25^{b}$ & $2.44^{b}$ & $13.40^{b}$ & Unpolluted \\
\hline & $4 \mathrm{~T} 2$ & 8 & 2.35 & $23.01^{\mathrm{b}}$ & 74.64 & 0.89 & $0.89^{c}$ & $0.24^{b}$ & $3.71^{b}$ & $16.18^{b}$ & Unpolluted \\
\hline \multirow{8}{*}{3} & $1 \mathrm{~T} 1$ & 7.8 & 3.54 & 13.96 & $82.5^{\mathrm{a}}$ & 0.48 & $1.56^{\mathrm{a}}$ & $0.05^{a}$ & $31.20^{\mathrm{a}}$ & $22.57^{a}$ & $336.35^{a}$ \\
\hline & $1 \mathrm{~T} 2$ & 7.9 & 2.95 & 13.15 & $83.9^{a}$ & 0.48 & $1.53^{\mathrm{a}}$ & $0.05^{\mathrm{a}}$ & $30.70^{\mathrm{a}}$ & $22.06^{a}$ & $333.62^{\mathrm{a}}$ \\
\hline & $2 \mathrm{~T} 1$ & 8.2 & 8.2 & 31.9 & $62.15^{b}$ & 0.41 & $1.38^{\mathrm{ab}}$ & $0.1^{b}$ & $13.84^{b}$ & $18.88^{b}$ & $228.34^{b}$ \\
\hline & $2 \mathrm{~T} 2$ & 8 & 12.5 & 24.36 & $63.06^{\mathrm{b}}$ & 0.4 & $1.20^{\mathrm{ab}}$ & $0.15^{b}$ & $8.00^{\mathrm{b}}$ & $19.25^{b}$ & $104.63^{b}$ \\
\hline & 3T1 & 7.7 & 5.35 & 31.81 & $62.83^{b}$ & 0.47 & $0.84^{b c}$ & $0.17^{b c}$ & $4.96^{b c}$ & $17.10^{c}$ & $10.83^{c}$ \\
\hline & $3 \mathrm{~T} 2$ & 7.9 & 5 & 31.62 & $63.37^{b}$ & 0.25 & $0.92^{\mathrm{bc}}$ & $0.16^{b c}$ & $5.74^{b c}$ & $17.12^{c}$ & $28.95^{c}$ \\
\hline & 4T1 & 7.9 & 9.5 & 26.68 & $63.82^{b}$ & 0.23 & $0.69^{c}$ & $0.2^{c}$ & $3.46^{c}$ & $12.80^{d}$ & $0^{c}$ \\
\hline & $4 \mathrm{~T} 2$ & 7.9 & 4.1 & 41.93 & $53.97^{b}$ & 0.42 & $0.30^{c}$ & $0.25^{c}$ & $1.21^{\mathrm{c}}$ & $12.38^{d}$ & $0^{c}$ \\
\hline
\end{tabular}

Moisture, C, N, clay, silt and sand results are expressed in percentage, EC in $\mu \mathrm{S} / \mathrm{cm}$ and TPH in $\mathrm{mg} / \mathrm{kg}$.

Different letters indicate significant differences between the different zones (ANOVA followed by the Newman-Keuls test, $p<0.05$ )

Table 2. Correlation coefficients between soil physical-chemical properties and ecological indexes

\begin{tabular}{|c|c|c|c|c|c|c|c|c|c|c|c|c|c|}
\hline & \multicolumn{4}{|c|}{ Site 1} & \multicolumn{4}{|c|}{ Site 2} & \multicolumn{5}{|c|}{ Site 3} \\
\hline & $\mathrm{N}$ & $\mathrm{s}$ & $H^{\prime}$ & $J$ & $\mathrm{~N}$ & $\mathrm{~s}$ & $H^{\prime}$ & $\mathrm{J}$ & $\mathrm{N}$ & $\mathrm{s}$ & $H^{\prime}$ & $\mathrm{J}$ & TPH \\
\hline $\mathrm{pH}$ & $0.91^{*}$ & $0.78^{*}$ & $0.79^{*}$ & $-0.88^{\star}$ & 0.50 & 0.57 & 0.64 & 0.67 & -0.09 & -0.16 & 0.05 & 0.32 & 0.20 \\
\hline Clay & 0.36 & 0.26 & 0.29 & -0.41 & 0.09 & 0.11 & 0.19 & 0.30 & 0.20 & 0.04 & 0.20 & 0.15 & -0.32 \\
\hline Silt & -0.33 & -0.52 & -0.47 & 0.22 & $-0.93^{*}$ & -0.57 & $-0.72^{*}$ & $-0.82^{*}$ & $0.77^{*}$ & $0.93^{*}$ & $0.88^{*}$ & 0.16 & $-0.79^{*}$ \\
\hline Sand & -0.04 & 0.13 & 0.08 & 0.14 & $0.89^{*}$ & 0.53 & 0.65 & 0.71 & $-0.79^{*}$ & $-0.88^{*}$ & $-0.86^{*}$ & -0.16 & $0.85^{\star}$ \\
\hline EC & -0.45 & -0.33 & -0.36 & 0.36 & 0.69 & 0.23 & 0.42 & 0.57 & -0.54 & -0.28 & -0.33 & 0.01 & 0.59 \\
\hline C & $-0.71^{*}$ & $-0.75^{\star}$ & $-0.85^{\star}$ & 0.54 & -0.19 & -0.50 & -0.61 & -0.65 & $-0.96^{*}$ & $-0.92^{*}$ & -0.64 & 0.32 & $0.90^{*}$ \\
\hline $\mathrm{N}$ & 0.70 & 0.70 & 0.63 & -0.69 & $0.87^{\star}$ & 0.11 & 0.26 & 0.40 & $0.95^{\star}$ & $0.90^{*}$ & 0.68 & -0.24 & $-0.94^{*}$ \\
\hline $\mathrm{C} / \mathrm{N}$ & $-0.92^{*}$ & $-0.84^{*}$ & $-0.90^{*}$ & $0.82^{*}$ & $-0.83^{*}$ & -0.66 & $-0.79^{*}$ & $-0.83^{*}$ & $-0.85^{*}$ & $-0.88^{*}$ & $-0.84^{*}$ & -0.08 & $0.97^{\star}$ \\
\hline$M$ & $-0.71^{*}$ & $-0.82^{*}$ & $-0.75^{\star}$ & 0.64 & $-0.88^{*}$ & -0.46 & -0.61 & $-0.73^{*}$ & -1.00 & $-0.86^{*}$ & -0.61 & 0.33 & $0.88^{*}$ \\
\hline TPH & X & X & X & $x$ & X & X & X & $x$ & $-0.87^{\star}$ & $-0.88^{*}$ & $-0.78^{*}$ & 0.03 & 1.00 \\
\hline
\end{tabular}

*Significant correlations ( $p<0.05)$; M: Moisture 
Table 3. Total species abundance $(N)$, species richness (S), Shannon-Weaver diversity $\left(H^{\prime}\right)$ and Pielou's evenness $(J)$ indexes in the three sites

\begin{tabular}{|c|c|c|c|c|c|c|c|c|c|c|c|c|c|c|c|c|c|}
\hline \multirow{2}{*}{ Sites } & \multirow{2}{*}{ Zones } & \multicolumn{4}{|c|}{$\mathrm{N}$} & \multicolumn{4}{|c|}{$S$} & \multicolumn{4}{|c|}{$\mathrm{H}^{\prime}$} & \multicolumn{4}{|c|}{$\mathrm{J}$} \\
\hline & & S1 & S2 & S3 & S4 & S1 & S2 & S3 & S4 & S1 & S2 & S3 & S4 & $\mathrm{S} 1$ & S2 & S3 & S4 \\
\hline \multirow{8}{*}{1} & $1 \mathrm{~T} 1$ & $10^{a}$ & $3^{a}$ & $11^{\mathrm{a}}$ & $7^{a}$ & 3 & $1^{a}$ & 5 & $4^{a}$ & 1.49 & $0.00^{\mathrm{a}}$ & 1.97 & $1.84^{\mathrm{a}}$ & $0.94^{\mathrm{a}}$ & $0.00^{\mathrm{a}}$ & $0.85^{a}$ & $0.92^{a}$ \\
\hline & $1 \mathrm{~T} 2$ & $8^{a}$ & $4^{a}$ & $12^{\mathrm{a}}$ & $7^{\mathrm{a}}$ & 3 & $1^{a}$ & 6 & $4^{a}$ & 1.50 & $0.00^{a}$ & 2.13 & $1.84^{\mathrm{a}}$ & $0.95^{\mathrm{a}}$ & $0.00^{\mathrm{a}}$ & $0.82^{a}$ & $0.92^{a}$ \\
\hline & $2 \mathrm{~T} 1$ & $24^{b}$ & $23^{b}$ & $72^{b}$ & $50^{b}$ & 6 & $3^{b}$ & 10 & $7^{b}$ & 2.18 & $1.40^{\mathrm{b}}$ & 2.52 & $2.43^{b}$ & $0.84^{\mathrm{ab}}$ & $0.89^{b}$ & $0.76^{a b}$ & $0.86^{b}$ \\
\hline & $2 \mathrm{~T} 2$ & $25^{b}$ & $30^{b}$ & $64^{b}$ & $65^{b}$ & 5 & $4^{b}$ & 9 & $10^{b}$ & 1.85 & $1.67^{b}$ & 2.60 & $2.69^{b}$ & $0.80^{\mathrm{ab}}$ & $0.83^{b}$ & $0.82^{a b}$ & $0.81^{b}$ \\
\hline & 3T1 & $31^{c}$ & $31^{\mathrm{b}}$ & $89^{c}$ & $77^{c}$ & 5 & $6^{c}$ & 9 & $13^{c}$ & 1.57 & $1.85^{b}$ & 2.39 & $2.97^{b}$ & $0.68^{b c}$ & $0.71^{b}$ & $0.75^{a b}$ & $0.80^{b}$ \\
\hline & 3T2 & $30^{c}$ & $47^{b}$ & $80^{c}$ & $83^{c}$ & 5 & $6^{c}$ & 10 & $10^{c}$ & 1.69 & $2.05^{b}$ & 2.55 & $2.50^{\mathrm{b}}$ & $0.73^{b c}$ & $0.79^{b}$ & $0.77^{a b}$ & $0.75^{b}$ \\
\hline & 4T1 & $35^{d}$ & $39^{b}$ & $81^{c}$ & $78^{c}$ & 7 & $9^{d}$ & 8 & $13^{d}$ & 1.99 & $2.94^{\mathrm{c}}$ & 2.08 & $2.93^{b}$ & $0.71^{c}$ & $0.93^{b}$ & $0.69^{b}$ & $0.79^{b}$ \\
\hline & $4 \mathrm{~T} 2$ & $36^{d}$ & $38^{b}$ & $86^{c}$ & $76^{c}$ & 5 & $8^{d}$ & 12 & $13^{d}$ & 1.40 & $2.45^{c}$ & 2.62 & $3.02^{b}$ & $0.60^{c}$ & $0.82^{b}$ & $0.73^{b}$ & $0.82^{b}$ \\
\hline \multirow{8}{*}{2} & 1T1 & $17^{a}$ & $1^{a}$ & $12^{\mathrm{a}}$ & $19^{a}$ & 4 & $1^{a}$ & $5^{a}$ & $3^{a}$ & $1.97^{a b}$ & $0.00^{\mathrm{a}}$ & 2.19 & $0.95^{\mathrm{a}}$ & $0.99^{a}$ & $0.00^{\mathrm{a}}$ & 0.94 & $0.60^{a}$ \\
\hline & $1 \mathrm{~T} 2$ & $15^{\mathrm{a}}$ & $1^{\mathrm{a}}$ & $8^{a}$ & $14^{a}$ & 4 & $1^{a}$ & $3^{a}$ & $3^{a}$ & $1.83^{a b}$ & $0.00^{a}$ & 1.06 & $0.73^{\mathrm{a}}$ & $0.91^{a}$ & $0.00^{a}$ & 0.67 & $0.46^{a}$ \\
\hline & 2T1 & $40^{a b}$ & $72^{b}$ & $38^{b}$ & $47^{b}$ & 5 & $6^{b}$ & $7^{b}$ & $9^{b}$ & $2.01^{\mathrm{ab}}$ & $1.43^{b}$ & 1.79 & $2.56^{b}$ & $0.86^{b}$ & $0.55^{b}$ & 0.64 & $0.81^{b}$ \\
\hline & $2 \mathrm{~T} 2$ & $31^{\mathrm{ab}}$ & $57^{b}$ & $30^{b}$ & $40^{b}$ & 7 & $5^{b}$ & $7^{b}$ & $10^{b}$ & $2.41^{\mathrm{ab}}$ & $1.33^{b}$ & 2.03 & $2.82^{b}$ & $0.86^{b}$ & $0.57^{b}$ & 0.72 & $0.85^{b}$ \\
\hline & 3T1 & $53^{b}$ & $33^{b}$ & $51^{c}$ & $61^{\mathrm{b}}$ & 6 & $3^{c}$ & $7^{b}$ & $13^{c}$ & $2.09^{a}$ & $1.45^{\mathrm{b}}$ & 1.97 & $3.09^{b}$ & $0.81^{b}$ & $0.92^{\mathrm{b}}$ & 0.70 & $0.83^{b}$ \\
\hline & 3T2 & $35^{b}$ & $66^{b}$ & $46^{c}$ & $52^{b}$ & 7 & $4^{c}$ & $8^{b}$ & $12^{c}$ & $2.28^{a}$ & $1.09^{b}$ & 2.33 & $3.03^{b}$ & $0.81^{b}$ & $0.54^{b}$ & 0.78 & $0.84^{b}$ \\
\hline & $4 \mathrm{~T} 1$ & $55^{b}$ & $78^{b}$ & $56^{c}$ & $73^{c}$ & 6 & $4^{c}$ & $3^{a}$ & $6^{d}$ & $1.50^{b}$ & $1.23^{b}$ & 1.06 & $1.99^{c}$ & $0.58^{c}$ & $0.61^{b}$ & 0.67 & $0.77^{b}$ \\
\hline & $4 \mathrm{~T} 2$ & $55^{b}$ & $78^{b}$ & $57^{c}$ & $85^{c}$ & 6 & $4^{c}$ & $4^{a}$ & $7^{d}$ & $1.57^{b}$ & $1.23^{b}$ & 1.07 & $2.19^{c}$ & $0.61^{c}$ & $0.61^{b}$ & 0.54 & $0.78^{b}$ \\
\hline \multirow{8}{*}{3} & 1T1 & $24^{a}$ & $10^{\mathrm{a}}$ & $10^{a}$ & $9^{a}$ & 4 & 4 & 3 & $3^{a}$ & $1.87^{\mathrm{ab}}$ & 1.90 & 1.49 & $0.99^{a}$ & $0.94^{\mathrm{a}}$ & $0.95^{\mathrm{a}}$ & 0.94 & $0.62^{\mathrm{a}}$ \\
\hline & $1 \mathrm{~T} 2$ & $32^{a}$ & $9^{a}$ & $3^{a}$ & $13^{a}$ & 5 & 3 & 2 & $3^{a}$ & $1.98^{\mathrm{ab}}$ & 1.53 & 0.92 & $0.99^{a}$ & $0.85^{a}$ & $0.97^{a}$ & 0.92 & $0.63^{a}$ \\
\hline & $2 \mathrm{~T} 1$ & $29^{a}$ & $29 \mathrm{ab}$ & $36^{a}$ & $32^{b}$ & 6 & 6 & 8 & $6^{\mathrm{ab}}$ & $2.05^{a}$ & 2.05 & 2.04 & $1.94^{b}$ & $0.79^{a}$ & $0.79^{b}$ & 0.68 & $0.75^{b}$ \\
\hline & $2 \mathrm{~T} 2$ & $36^{a}$ & $29^{\mathrm{ab}}$ & $16^{a}$ & $33^{b}$ & 5 & 7 & 6 & $5^{\mathrm{ab}}$ & $2.04^{a}$ & 2.00 & 2.15 & $1.58^{b}$ & $0.88^{a}$ & $0.71^{b}$ & 0.83 & $0.68^{b}$ \\
\hline & 3T1 & $44^{b}$ & $55^{b}$ & $38^{a}$ & $51^{c}$ & 6 & 5 & 6 & $9^{b}$ & $1.62^{b}$ & 1.99 & 1.85 & $2.24^{b}$ & $0.62^{b}$ & $0.85^{a}$ & 0.72 & $0.71^{b}$ \\
\hline & 3T2 & $47^{b}$ & $35^{b}$ & $30^{a}$ & $45^{c}$ & 5 & 3 & 2 & $7^{b}$ & $1.58^{b}$ & 1.42 & 1.00 & $2.07^{b}$ & $0.68^{b}$ & $0.90^{\mathrm{a}}$ & 1.00 & $0.74^{b}$ \\
\hline & 4T1 & $58^{c}$ & $74^{c}$ & $63^{b}$ & $83^{d}$ & 5 & 4 & 3 & $7^{b}$ & $1.47^{b}$ & 1.28 & 0.82 & $1.59^{\mathrm{b}}$ & $0.63^{b}$ & $0.64^{c}$ & 0.51 & $0.57^{a}$ \\
\hline & $4 \mathrm{~T} 2$ & $60^{c}$ & $72^{c}$ & $59^{b}$ & $86^{d}$ & 6 & 4 & 4 & $10^{b}$ & $1.74^{b}$ & 1.14 & 1.05 & $1.93^{b}$ & $0.67^{b}$ & $0.57^{c}$ & 0.53 & $0.58^{a}$ \\
\hline
\end{tabular}

S1: November sampling; S2: January sampling; S3: March sampling; S4: May sampling

Different letters indicate significant differences between the different zones (ANOVA followed by Newman-Keuls test, $p<0.05$ ).

Galactites tomentosa and Scolymus hispanicus avoided the contaminated zones. The first lot of species tolerated the contamination and could represent potential candidates for the phytoremediation of hydrocarbon contaminated sites. Xanthium strumarium, a plant species which is able to grow in many habitats and conditions (Weaver and Lechowicz, 1983; Saric et al., 2012), preferred zones 1, where the pollution is most important; consequently, this plant species could have a good capacity to establish in hydrocarbon contaminated sites. Besides, Gulezian et al. (2012) reported the tolerance of
Conium maculatum to the metals and PAHs contamination. The second lot of species is potentially susceptible to hydrocarbon contamination. Xanthium strumarium, Avena sp, Conium maculatum and Oxalis pes-caprae are weed plant species, considered in some parts of the world as invasive species (Castro et al., 2007; Nakova et al., 2004; Sharma et al., 2010; Vetter, 2004). Hierro et al. (2006) suggested that human activities could increase species invasions; therefore, it is possible that the changes caused by the contamination helped in the establishment of the weed species. 
Table 4. Phytosociological table of site 1 with species abundance-dominance

\begin{tabular}{|c|c|c|c|c|c|c|c|c|c|c|c|c|c|c|c|c|c|c|c|c|c|c|c|c|c|c|c|c|c|c|c|c|c|}
\hline \multirow[b]{3}{*}{ Zones } & \multicolumn{8}{|c|}{ November } & \multicolumn{8}{|c|}{ January } & \multicolumn{8}{|c|}{ March } & \multicolumn{9}{|c|}{ Mai } \\
\hline & \multicolumn{4}{|c|}{ Transect 1} & \multicolumn{4}{|c|}{ Transect 2} & \multicolumn{4}{|c|}{ Transect 1} & \multicolumn{4}{|c|}{ Transect 2} & \multicolumn{4}{|c|}{ Transect 1} & \multicolumn{4}{|c|}{ Transect 2} & \multicolumn{5}{|c|}{ Transect 1} & \multicolumn{4}{|c|}{ Transect 2} \\
\hline & 1 & 2 & 3 & 4 & 1 & 2 & 3 & 4 & 1 & 2 & 3 & 4 & 1 & 2 & 3 & 4 & 1 & 2 & 3 & 4 & 1 & 2 & 3 & 4 & 1 & 2 & 3 & 4 & & 1 & 2 & 3 & 4 \\
\hline Avena sp & 1 & 2 & 2 & 1 & 1 & 2 & 2 & 1 & 1 & 3 & 3 & 2 & 1 & 3 & 3 & 2 & + & 3 & 3 & 3 & + & 3 & 3 & 3 & + & 3 & 3 & 3 & & 1 & 3 & 3 & 3 \\
\hline $\begin{array}{c}\text { Ampelodesmos } \\
\text { mauritanicus }\end{array}$ & & & & & & & & & & & & & & & & & & 1 & & & & 1 & & & & & 2 & 2 & & & & 2 & 2 \\
\hline $\begin{array}{l}\text { Anogramma } \\
\text { leptophylla }\end{array}$ & & & & & & & & & & & & & & & & & & & & & & & & + & & & & & & & & & 1 \\
\hline Daucus carota & & & & & & & & & & & & & & & & & & & & & & & & & & & + & + & & & & + & + \\
\hline $\begin{array}{l}\text { Centaurium } \\
\text { spicatum }\end{array}$ & & + & & & & + & & & & & & & & & & + & & + & + & & & + & & & & & & & & & & & \\
\hline Cerinthe major & & + & & 1 & & & & 1 & & 1 & + & 1 & & 1 & + & 1 & & 1 & 1 & & & 1 & & + & & 1 & + & 1 & & & 1 & & \\
\hline Conium maculatum & 1 & 1 & 1 & & 1 & 1 & 1 & & & 2 & 2 & 2 & & 1 & 2 & 1 & 1 & 2 & 2 & 2 & + & 2 & 2 & 2 & & 2 & 2 & 2 & & & 2 & 2 & 2 \\
\hline Foeniculum vulgare & & & & & & & & & & & & & & & & & & & & & & & + & & & & & & & & & & \\
\hline $\begin{array}{c}\text { Fraxinus } \\
\text { angustifolia }\end{array}$ & & & & & & & & & & & & & & & & & 1 & + & 1 & & 1 & 1 & 1 & & 1 & 1 & 1 & & & 1 & 1 & & \\
\hline $\begin{array}{l}\text { Galactites } \\
\text { tomentosa }\end{array}$ & & & & 1 & & & & & & & 1 & 1 & & & 2 & 1 & & & & + & & & & 1 & & & & 1 & & & & & 1 \\
\hline $\begin{array}{l}\text { Geranium } \\
\text { robertanium }\end{array}$ & & & + & 1 & & & 1 & 1 & & & & & & & & + & & & & & + & & & + & & & & 1 & & & & & \\
\hline Lathyrus sp & & & & & & & & & & & & & & & & & & & & & & & & & 1 & 1 & 1 & & & 1 & 1 & 1 & + \\
\hline Leontodon sp & & & & & & & & & & & & & & & & & & & & & & & 1 & & & & + & & & & & 1 & \\
\hline Malva sylvestris & & & & 1 & & & & & & & & + & & & & 1 & & & & + & & & & + & & & & 1 & & & & & 1 \\
\hline Medicago sp & & & & & & & & & & & & & & & & & & + & 1 & & & & 1 & + & & & + & + & & & 1 & & \\
\hline Oxalis pes-caprae & 1 & 2 & 2 & 4 & 1 & 1 & 2 & 4 & & 1 & 2 & 3 & & & + & 3 & & 1 & 1 & 3 & & 1 & + & 3 & & 1 & & + & & & 1 & & 1 \\
\hline Plantago lanceolata & & & & & & & & & & & & & & & & & & & & & & & & + & & & 1 & & & & 1 & + & \\
\hline Rubus fruticosus & & & & & & & & & & & & & & & & & & & & + & & & & & & & + & 1 & & & & 1 & 1 \\
\hline Senecio vulgaris & & & 1 & 1 & & & 1 & + & & & + & + & & & + & 1 & & & & & & & + & & & 1 & 2 & + & & & 1 & 2 & 1 \\
\hline Sonchus oleraceus & & & & & & & & & & & & & & & & & & 1 & 2 & 1 & & + & 2 & 2 & & & & & & & & & 1 \\
\hline Taraxacum sp & & & & & & & & & & & & & & & & & + & & & & + & & & & & & & & & & & & \\
\hline $\begin{array}{c}\text { Trifolium } \\
\text { tomentosum }\end{array}$ & & & & & & & & & & & & & & & & & & & & & & & & & & & 1 & 2 & & & 1 & 1 & 2 \\
\hline Vicia sativa & & 1 & & & & + & & & & & & + & & & & & + & + & + & 1 & + & 1 & 1 & 1 & & & & & & & & & \\
\hline $\begin{array}{l}\text { Xanthium } \\
\text { strumarium }\end{array}$ & & & & & & & & & & & & & & & & & & & & & & & & & 1 & & & & & + & & & \\
\hline
\end{tabular}

+: range of cover $<5 \%$ and few individuals; 1 : range of cover $<5 \%$ and numerous individuals; 2: range of cover 5-25\%; 3: range of cover $25-50 \%$; 4 : range of cover $50-75 \%$; 5 : range of cover $75-100 \%$. 
Table 5. Phytosociological table of site 2 with species abundance-dominance

\begin{tabular}{|c|c|c|c|c|c|c|c|c|c|c|c|c|c|c|c|c|c|c|c|c|c|c|c|c|c|c|c|c|c|c|c|c|}
\hline \multirow[b]{3}{*}{ Zones } & \multicolumn{8}{|c|}{ November } & \multicolumn{8}{|c|}{ January } & \multicolumn{8}{|c|}{ March } & \multicolumn{8}{|c|}{ Mai } \\
\hline & \multicolumn{4}{|c|}{ Transect 1} & \multicolumn{4}{|c|}{ Transect 2} & \multicolumn{4}{|c|}{ Transect 1} & \multicolumn{4}{|c|}{ Transect 2} & \multicolumn{4}{|c|}{ Transect 1} & \multicolumn{4}{|c|}{ Transect 2} & \multicolumn{4}{|c|}{ Transect 1} & \multicolumn{4}{|c|}{ Transect 2} \\
\hline & 1 & 2 & 3 & 4 & 1 & 2 & 3 & 4 & 1 & 2 & 3 & 4 & 1 & 2 & 3 & 4 & 1 & 2 & 3 & 4 & 1 & 2 & 3 & 4 & 1 & 2 & 3 & 4 & 1 & 2 & 3 & 4 \\
\hline Anagallis moneli & & & & & & & & & & & & & & & & & & + & + & & & + & + & & & & & & & & & \\
\hline Avena sp & 1 & 2 & 2 & 3 & 1 & 2 & 2 & 3 & & 3 & 4 & 4 & & 3 & 3 & 4 & 1 & 3 & 4 & 4 & & 2 & 4 & 4 & & 2 & 2 & 4 & & 2 & 2 & 4 \\
\hline Beta vulgaris & & & 1 & + & & & + & + & & & & + & & & & + & & & & & & & & & & & & & & & & \\
\hline Bromus sp & & & & & & & & & & & & & & & & & & & & & & & & & + & 1 & 1 & 2 & + & 1 & 1 & 2 \\
\hline Bromus sp & & & & & & & & & & & & & & & & & & & & & & & & & & & & 1 & & & & 1 \\
\hline $\begin{array}{c}\text { Chenopodium } \\
\text { album }\end{array}$ & & & & & & & & & & & & & & & & & & & & & & & & & & 2 & 2 & & & 2 & 2 & \\
\hline Conium maculatum & 1 & 1 & 1 & + & 1 & 1 & 1 & 1 & & 1 & 1 & & & 1 & 1 & & + & 1 & + & & + & 1 & + & & + & 1 & 1 & & + & 1 & 1 & \\
\hline Elymus repens & & & & & & & & & & & & & & & & & & & & & & & & & & + & & & & 1 & & \\
\hline Fumaria capreolata & & & & & & & & & & & & & & & & & & & 1 & & & & & & & & & & & & & \\
\hline $\begin{array}{l}\text { Galactites } \\
\text { tomentosa }\end{array}$ & & & + & + & & + & + & + & & + & + & & & + & + & & & + & + & + & & + & + & + & & + & + & + & & + & + & + \\
\hline Hordeum murinum & & & & & & & & & & & & & & & & & & & & & & & & & & & + & & & & & \\
\hline Inula viscosa & & & & + & & & & + & & 1 & & & & & & & & 1 & & & & & & & & & & & & & & \\
\hline Lamium sp & & & & & & & & & & & & & & + & & & + & & & & & & & & & & & & & & & \\
\hline Lolium perenne & & & & & & & & & & & & & & & & & & & & & & & & & & & & 1 & & & & 1 \\
\hline Lysimachia sp & & & & & & + & & & & & & & & & & & & & & & & + & + & & & + & + & & & + & + & \\
\hline Malva sylvestris & & 1 & 2 & & & 1 & 1 & & & & & & & & & & & & + & & & & & & & & + & & & & 1 & \\
\hline Medicago sp & & & & & & & & & & & & & & & & & & & & & & & & & & + & + & & & + & + & 1 \\
\hline Oxalis pes-caprae & 1 & 2 & 2 & 3 & + & 2 & 2 & 3 & & 2 & 2 & 3 & & 2 & 4 & 3 & + & 2 & 3 & 3 & + & 2 & 3 & 3 & & & 1 & & & + & + & \\
\hline Plantago lanceolata & & & & & & & + & & & & & & & & & & & & & & & & & & & & & & & & & \\
\hline $\begin{array}{l}\text { Scolymus } \\
\text { hispanicus }\end{array}$ & & & & & & & & & & & & + & & & & + & & & & & & & & + & & & & 1 & & & & 1 \\
\hline Senecio vulgaris & & & & & & & & & & + & & & & & & & & + & & & & + & + & & & & 1 & & & & 1 & \\
\hline Sonchus oleraceus & & 2 & & & & + & & & & & & & & & & & & & & & & & + & & & & 1 & & & & 1 & \\
\hline $\begin{array}{l}\text { Xanthium } \\
\text { strumarium }\end{array}$ & 1 & & & & 2 & & & & + & & & & + & & & & 1 & & & & 1 & & & & 2 & 1 & + & & 2 & + & + & \\
\hline
\end{tabular}

+: range of cover $<5 \%$ and few individuals; 1: range of cover < 5\% and numerous individuals; 2 : range of cover 5-25\%; 3: range of cover $25-50 \%$; 4: range of cover 50-75\%; 5: range of cover $75-100 \%$. 
Table 6. Phytosociological table of site 3 with species abundance-dominance

\begin{tabular}{|c|c|c|c|c|c|c|c|c|c|c|c|c|c|c|c|c|c|c|c|c|c|c|c|c|c|c|c|c|c|c|c|c|}
\hline \multirow[b]{3}{*}{ Zones } & \multicolumn{8}{|c|}{ November } & \multicolumn{8}{|c|}{ January } & \multicolumn{8}{|c|}{ March } & \multicolumn{8}{|c|}{ Mai } \\
\hline & \multicolumn{4}{|c|}{ Transect 1} & \multicolumn{4}{|c|}{ Transect 2} & \multicolumn{4}{|c|}{ Transect 1} & \multicolumn{4}{|c|}{ Transect 2} & \multicolumn{4}{|c|}{ Transect 1} & \multicolumn{4}{|c|}{ Transect 2} & \multicolumn{4}{|c|}{ Transect 1} & \multicolumn{4}{|c|}{ Transect 2} \\
\hline & 1 & 2 & 3 & 4 & 1 & 2 & 3 & 4 & 1 & 2 & 3 & 4 & 1 & 2 & 3 & 4 & 1 & 2 & 3 & 4 & 1 & 2 & 3 & 4 & 1 & 2 & 3 & 4 & 1 & 2 & 3 & 4 \\
\hline Anagallis moneli & & & & & & & & & & & 1 & & & & & & & & 1 & & & & & & & & + & & & & & \\
\hline Andryala integrifolia & & & & & & & & & & & & & & & & & & & & & & & & & & & & & & & & + \\
\hline Avena sp & 2 & 1 & 2 & 3 & 2 & 2 & 2 & 3 & 1 & 2 & 3 & 4 & + & 2 & 3 & 4 & 1 & 1 & 3 & 5 & & + & 2 & 5 & + & + & 3 & 3 & & + & 3 & 3 \\
\hline Beta vulgaris & & & & + & & & & 1 & & & & + & & & & + & & & & & & & & & & & & & & & & \\
\hline Bromus sp & & & & & & & & & & & & & & & & & & & & & & & & & + & + & 2 & 4 & & + & 2 & 4 \\
\hline Bromus sp & & & & & & & & & & & & & & & & & & & & & & & & & & & & 1 & & & & 1 \\
\hline Conium maculatum & 1 & + & + & & 1 & 1 & 1 & & 1 & 1 & 2 & & 1 & 1 & 1 & & & + & 1 & & & + & & & & & + & & + & & & \\
\hline Echium creticum & & & & & & & & & & & & & & & & & & & & & & & & & & & & & & & & + \\
\hline Elymus repens & & 2 & + & + & & 3 & & 1 & & 2 & & & & 3 & & & & 1 & & & & 1 & & & & 3 & 1 & 1 & & 3 & 1 & 1 \\
\hline Fumaria capreolata & & & & & & & & & & & & & & + & & & & & & & & & & & & & & & & & & \\
\hline $\begin{array}{l}\text { Galactites } \\
\text { tomentosa }\end{array}$ & & & & & & & & & & & & & & & & & & & & + & & & & 1 & & & & & & & & \\
\hline Hordeum murinum & & & & & & & & & & & & & & & & & & & & & & & & & & + & 1 & 1 & & & 1 & 1 \\
\hline Inula viscosa & & & & & + & & & & & & & & & & & & & & & & & & & & & & & & & & & \\
\hline Lolium perenne & & & & & & & & & & & & & & & & & & & & & & & & & & + & & & + & + & & \\
\hline Malva sylvestris & & + & + & + & & 1 & + & + & & + & + & & & + & & & & + & & & & + & & + & & & & & & & & + \\
\hline Oxalis pes-caprae & 1 & 1 & 3 & 3 & 1 & 1 & 4 & 3 & + & & 4 & 3 & & & 4 & 3 & + & & 3 & 2 & + & & 4 & 2 & & & & & & & & \\
\hline Phragmites australis & & & & & & & & & & & & & & & & & & & & & & & & & & & & + & & & & + \\
\hline Plantago lanceolata & & & & & & & & + & & & & & & & & & & & & & & & & & & & & & & & & \\
\hline Poa annua & & & & & & & & & & & & & & & & & & + & & & & & & & & & & & & & & \\
\hline $\begin{array}{l}\text { Scolymus } \\
\text { hispanicus }\end{array}$ & & & & & & & & & & & & 2 & & & & 1 & & & & & & & & & & & + & + & & & + & + \\
\hline Senecio vulgaris & & & & & & & & & & + & & & & & & & & + & + & & & & & & & & & & & & & \\
\hline Sonchus oleraceus & & + & + & & & & + & & & 1 & & & & 1 & & & & + & + & & & + & & & & & & & & & & \\
\hline $\begin{array}{l}\text { Trifolium tomento- } \\
\text { sum }\end{array}$ & & & & & & & & & & & & & & & & & & & & & & & & & & & + & & & & + & \\
\hline $\begin{array}{l}\text { Xanthium } \\
\text { strumarium }\end{array}$ & 2 & & & & 2 & & & & 2 & & & & 2 & + & & & 1 & 1 & & & + & 3 & & & 2 & 2 & + & & 2 & 2 & + & \\
\hline
\end{tabular}

+: range of cover < 5\% and few individuals; 1 : range of cover < 5\% and numerous individuals; 2: range of cover 5-25\%; 3: range of cover $25-50 \%$; 4 : range of cover $50-75 \%$; 5 : range of cover $75-100 \%$. 
We noticed low $\mathrm{H}^{\prime}$ values in zones 1, corresponding to low abundances and low species richness. Sites 2 and 3 presented low H' values in zones 4, and in zones 3 for site 3 (Table 3), due mainly to the Avena sp and Oxalis pes-caprae dominance. Low H' values in zones 4 of site 1 are due to the Oxalis pes-caprae dominance.
The Sorensen-Dice coefficient showed that the highest similarities were between zones 2 and 3 , and zones 3 and 4 . The highest differences were between zones 1 and 4, and zones 2 and 4 (Figure 3). Thus, there is a clear differentiation between the more polluted zones and the less polluted and unpolluted zones.

Fig. 3. The Sorensen-Dice coefficient (DSC) values of the vegetation sampling in the three sites (Transects 1 and 2)

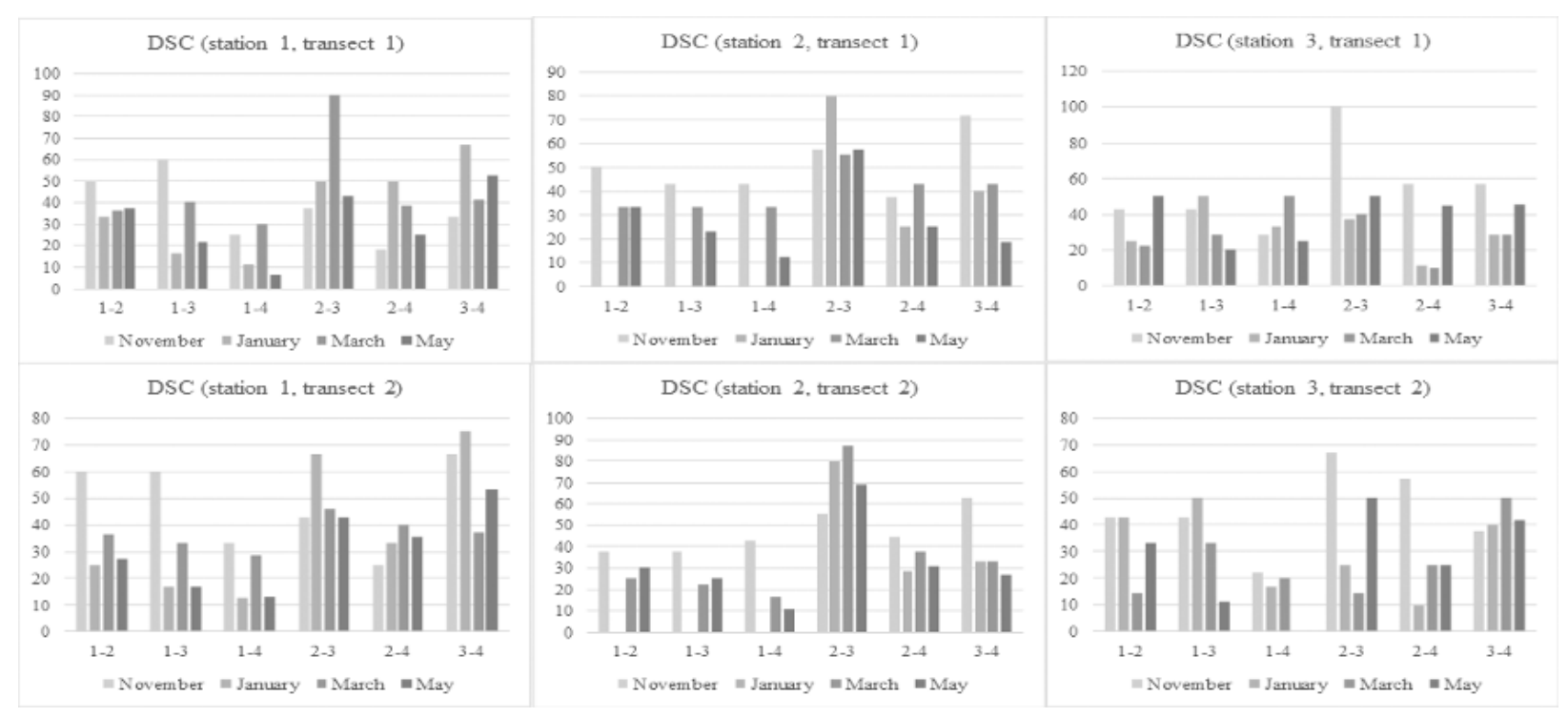

Pearson linear correlation (Table 2) revealed that the $\mathrm{C} / \mathrm{N}$ ratio, moisture and TPH are the parameters influencing mainly the different ecological indexes. For site $1, \mathrm{pH}$ is also an important parameter. The abundance and $\mathrm{H}^{\prime}$ seem to be the most influenced indexes; thus, there are no significant correlations for $\mathrm{S}$ in site 2 and for $\mathrm{J}$ in site 3 . Consequently, the vegetation structure was influenced by the contamination and the changes in soil properties.

Concerning soil invertebrates, we identified 10 invertebrate orders, spread over the four zones (Table 7). Hymenoptera was the most abundant order throughout the different zones, followed by the Coleoptera and Araneae orders. The response of invertebrate species to a soil contamination depends on many factors, like the specific behaviour of each species under the stress caused by the pollution and its ability to tolerate it; hence, there is a differentiation between the invertebrate communities living in polluted and unpolluted soils (van Straalen, 2004). Therefore, we can differentiate two lots of invertebrates. The first lot is composed of Isopoda, Panpulmonata, Orthoptera, Diptera and Mantodea orders, which are mostly present in the most polluted zones. Hymenoptera abundances did not show a clear tendency; we observed the lowest abundances in zones 1 and the highest in zones 2. It was observed that Isopoda and Hymenoptera can take advantage of high levels of contamination (Faulkner and Lochmiller, 2000), and according to Gospodarek et al. (2019)survival and body mass change of P. scaber reared in investigated soils were observed. The delivered food was not contaminated with PDSs. P. scaber reveals a considerable resistance in a short (4 weeks, Isopoda could have good capacities for the biotransformation of petroleum-derived substances and a low vulnerability to the penetration 
of these substances through the integuments. Panpulmonata are known for their ability of bioaccumulation and are used as bioindicators (Oehlmann and Schulte-Oehlmann, 2003). In their study, García-Segura et al. (2018) reported a decrease of Gastropods populations with an increase in TPH levels. The second lot is composed by Parasitiformes, Hemiptera, Araneae and Coleoptera orders, which showed a preference for the less polluted and unpolluted zones. Rusin and
Gospodarek (2016) observed in their study that petroleum-based substances in most cases did not have a negative effect on the number of spiders; on the other hand, Gospodarek et al. (2019)survival and body mass change of P. scaber reared in investigated soils were observed. The delivered food was not contaminated with PDSs. P. scaber reveals a considerable resistance in a short ( 4 weeks observed a negative correlation between the Acari and some hydrocarbons.

Table 7. Invertebrate order abundance in each zone of each transect and total abundance for the orders

\begin{tabular}{c|c|c|c|c|c|c|c|c|c}
\hline Zones & $1 \mathrm{~T} 1$ & $1 \mathrm{~T} 2$ & $2 \mathrm{~T} 1$ & $2 \mathrm{~T} 2$ & $3 \mathrm{~T} 1$ & $3 \mathrm{~T} 2$ & $4 \mathrm{~T} 1$ & $4 \mathrm{~T} 2$ & Total \\
\hline Araneae & 13 & 7 & 12 & 12 & 21 & 17 & 21 & 18 & 121 \\
\hline Coleoptera & $4^{\mathrm{a}}$ & $10^{\mathrm{a}}$ & $19^{\mathrm{ab}}$ & $35^{\mathrm{ab}}$ & $56^{\mathrm{c}}$ & $73^{\mathrm{c}}$ & $41^{\mathrm{bc}}$ & $46^{\mathrm{bc}}$ & 284 \\
\hline Diptera & 2 & 5 & 6 & 6 & 1 & 4 & 2 & 4 & 30 \\
\hline Hemiptera & 3 & 2 & 2 & 8 & 11 & 12 & 5 & 10 & 53 \\
\hline Hymenoptera & 125 & 153 & 347 & 239 & 181 & 178 & 376 & 189 & 1788 \\
\hline Isopoda & 3 & 11 & 1 & 4 & 2 & 1 & 1 & 0 & 23 \\
\hline Mantodea & 0 & 1 & 1 & 0 & 0 & 0 & 0 & 0 & 2 \\
\hline Parasitiformes & 1 & 1 & 2 & 4 & 4 & 5 & 15 & 5 & 37 \\
\hline Orthoptera & 0 & 2 & 1 & 1 & 1 & 0 & 0 & 0 & 5 \\
\hline Panpulmonata & 1 & 0 & 4 & 1 & 0 & 0 & 0 & 1 & 7 \\
\hline
\end{tabular}

Different letters indicate significant differences between the different zones (ANOVA followed by the Newman-Keuls test, $p<0.05$ ).

Order abundance seems to be influenced by the soil contamination. There is a general tendency, showing a clear differentiation between zones 1 and the other zones. Zones 1 present the lowest abundance values (Table 8 ). The number of orders were not negatively influenced by the contamination; thus, zones 4 presented the lowest values (Table 8). However, when it comes to the species richness (Figure 4), there is an obvious tendency demonstrating that zones 1 are the poorest zones. $\mathrm{H}^{\prime}$ and $\mathrm{J}$ values present a general tendency, showing higher values for zones 3 and 4 compared with zones 1 and 2 (Table 8). Sorensen-Dice coefficient values for transect 1 did not show any tendency, but in transect 2, the lowest value was between zones 1 and zones 4 (Figure 5). Therefore, there is a differentiation between the most polluted zones and the less polluted and unpolluted zones. Furthermore, there are some other parameters to consider, as the relationships between the vegetation structure and the invertebrates. It was shown that some invertebrate groups like Coleoptera and phytophagous insects have a close relationship with vegetation structure and plant species diversity (Chenchouni et al., 2015). The distribution of some groups, as Coleoptera and Hemiptera individuals could be partly explained by the vegetation cover, which is more important in zones 4 , followed by zones 3,2 and 1, respectively. Araneae distribution could also be related to the Hemiptera distribution, since they are predators of these insects. So, invertebrate communities can be affected directly by the contamination and indirectly by the changes in the vegetation structure produced by the contamination. 
Table 8. Total species abundance ( $N$ ), number of orders (NO), Shannon-Weaver diversity $\left(H^{\prime}\right)$ and Pielou's evenness $(J)$ indexes in each zone of each transect

\begin{tabular}{c|c|c|c|c}
\hline Zones & $\mathrm{N}$ & $\mathrm{NO}$ & $\mathrm{H}^{\prime}$ & $\mathrm{J}$ \\
\hline $1 \mathrm{~T} 1$ & 152 & $8^{\mathrm{a}}$ & 1.07 & 0.36 \\
\hline $1 \mathrm{~T} 2$ & 192 & $8^{\mathrm{a}}$ & 1.24 & 0.41 \\
\hline $2 \mathrm{~T} 1$ & 395 & $10^{\mathrm{b}}$ & 0.82 & 0.25 \\
\hline $2 \mathrm{~T} 2$ & 310 & $9^{\mathrm{b}}$ & 1.28 & 0.40 \\
\hline $3 \mathrm{~T} 1$ & 277 & $8^{\mathrm{a}}$ & 1.53 & 0.51 \\
\hline $3 \mathrm{~T} 2$ & 290 & $7^{\mathrm{a}}$ & 1.57 & 0.56 \\
\hline $4 \mathrm{~T} 1$ & 461 & $7^{\mathrm{a}}$ & 1.03 & 0.37 \\
\hline $4 \mathrm{~T} 2$ & 273 & $7^{\mathrm{a}}$ & 1.45 & 0.52 \\
\hline
\end{tabular}

Different letters indicate significant differences between the different zones (ANOVA followed by the Newman-Keuls test, $p<0.05$ ).

Fig. 4. Sorensen-Dice coefficient (DSC) values of the invertebrates sampling (Transects 1 and 2)

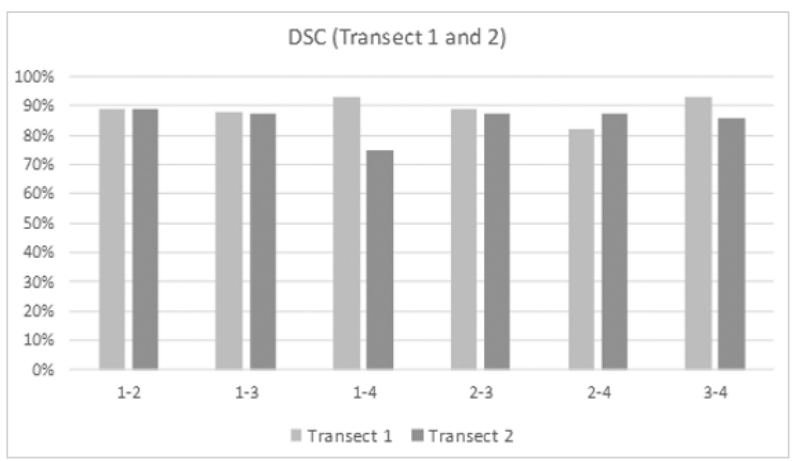

Fig. 5. Invertebrates number of species in each zone of each sampling. Different letters indicate significant differences between the different zones (ANOVA followed by the Newman-Keuls test, $p<0.05$ )

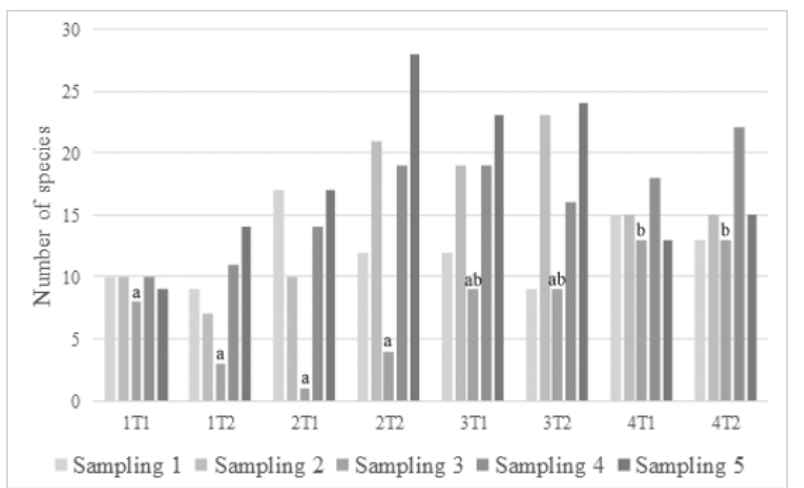

\section{Conclusions}

Petrol station wastes affected some soil properties as the TPH, N, C, pH and moisture. The vegetation and soil invertebrates were influenced as regards the abundance, the species richness, the biological diversity $\left(\mathrm{H}^{\prime}\right)$ and the species evenness $(\mathrm{J})$ by the contamination and the changes in soil properties. Some weed plant species showed a tolerance to the contamination, as Xanthium strumarium, Avena sp, Conium maculatum and Oxalis pes-caprae, and some invertebrate orders, as Isopoda, Panpulmonata and Orthoptera were mostly present in the contaminated zones.

\section{References}

Adam G. and Duncan H. (2002) Influence of diesel fuel on seed germination. Environmental Pollution 120(2): 363-370. https://doi.org/10.1016/S0269-7491(02)00119-7

Adam G. and Duncan H. (2003) The Effect of Diesel Fuel on Common Vetch (Vicia Sativa L.) Plants. Environmental Geochemistry and Health 25(1): 123-130. https://doi. org/10.1023/A:1021228327540

Akpan G.U. and Udoh B.T. (2013) Evaluation of Some Properties of Soils Affected by Diesel Oil Pollution in Uyo, Niger Delta Area, Nigeria. Journal of Biology, Agriculture and Healthcare 3(8): 33-42.

Alharbi B.H., Pasha M.J., Alhudhodi A.H. and Alduwais A.K. (2018) Assessment of soil contamination caused by underground fuel leakage from selected gas stations in Riyadh, Saudi Arabia. Soil and Sediment Contamination: An International Journal 27(8): 674-691. https://doi.org/10.1080/15320383.20 18.1503228

Borowiec M., Hoffmann K., Huculak M., Rogowski S. and Hoffmann J. (2008) Effect of petrol stations on the contamination of the soil environment. Czasopismo-Techniczne 2-Ch(16): 62-69.

Braun-Blanquet J., Conard H.S. and Fuller G.D. (1932) Plant Sociology the Study of Plant Communities. McGraw-Hill book company, inc. 439 p. https://doi.org/10.5962/bhl.title.7161

Castro S., Loureiro J., Santos C., Ater M., Ayensa G. and Navarro L. (2007) Distribution of Flower Morphs, Ploidy Level and Sexual Reproduction of the Invasive Weed Oxalis pes-caprae in the Western Area of the Mediterranean Region. Annals of Botany 99(3): 507-517. https://doi.org/10.1093/aob/mcl273 
Chenchouni H., Menasria T., Neffar S., Chafaa S., Bradai L., Chaibi R., Mekahlia M.N., Bendjoudi D. and Si Bachir D. (2015) Spatiotemporal diversity, structure and trophic guilds of insect assemblages in a semi-arid Sabkha ecosystem. PeerJ 3: e860. https://doi.org/10.7717/peerj.860

Dazy M., Béraud E., Cotelle S., Grévilliot F., Férard J.-F. and Masfaraud J.-F. (2009) Changes in plant communities along soil pollution gradients: responses of leaf antioxidant enzyme activities and phytochelatin contents. Chemosphere 77(3): 376-383. https://doi.org/10.1016/j.chemosphere.2009.07.021

Dice L.R. (1945) Measures of the Amount of Ecologic Association Between Species. Ecology 26(3): 297-302. https://doi. org/10.2307/1932409

Erstfeld K.M. and Snow-Ashbrook J. (1999) Effects of chronic low-level PAH contamination of soil invertebrate communities. Chemosphere 39(12): 2117-2139. https://doi.org/10.1016/ S0045-6535(98)00421-4

Fatokun K., Lewu FB., Zharare GE. (2015) Phyotoxicity of diesel soil contamination on the germination of Lactuca sativa and Ipomoea batatas. Journal of Environmental Biology 36(6): 1337-1344.

Faulkner B.C. and Lochmiller R.L. (2000) Increased Abundance of Terrestrial Isopod Populations in Terrestrial Ecosystems Contaminated with Petrochemical Wastes. Archives of Environmental Contamination and Toxicology, 39(1): 86-90. https://doi. org/10.1007/s002440010083

García-Segura D., Castillo-Murrieta IM., Martínez-Rabelo F., Gomez-Anaya A., Rodríguez-Campos J., Hernández-Castellanos B., Contreras-Ramos SM., Barois I. (2018) Macrofauna and mesofauna from soil contaminated by oil extraction. Geoderma 332: 180-189. https://doi.org/10.1016/j.geoderma.2017.06.013

Gospodarek J., Petryszak P., Kołoczek H., Rusin M. (2019) The effect of soil pollution with petroleum-derived substances on Porcellio scaber Latr. (Crustacea, Isopoda). Environmental Monitoring and Assessment 191(38). https://doi.org/10.1007/ s10661-018-7181-6

Grzes' I.M. (2009) Ant species richness and evenness increase along a metal pollution gradient in the Bolesław zinc smelter area. Pedobiologia 53(1): 65-73. https://doi.org/10.1016/j.pedobi.2009.03.002

Gulezian P.Z., Ison J.L. and Granberg K.J. (2012) Establishment of an Invasive Plant Species (Conium maculatum) in Contaminated Roadside Soil in Cook County, Illinois. The American Midland Naturalist 168(2): 375-395. https://doi.org/10.1674/00030031-168.2.375

Henry J.A. (1998) Composition and toxicity of petroleum products and their additives. Human \& Experimental Toxicology 17(2): 111-123. https://doi.org/10.1177/096032719801700206
Hierro J.L., Villarreal D., Eren O., Graham J.M. and Callaway R.M. (2006) Disturbance Facilitates Invasion: the Effects are Stronger Abroad than at Home. The American Naturalist 168(2): 144156. https://doi.org/10.1086/505767

Kathi S. and Khan AB. (2011) Phytoremediation Approaches to PAH Contaminated Soil. Indian Journal of Science and Technology 4(1): 56-63-63. https://doi.org/10.17485/ijst/2011/ v4i1/29935

Luhach J. and Chaudhry S. (2012) Effect of Diesel Fuel Contamination on Seed Germination and Growth of Four Agricultural Crops. Universal Journal of Environmental Research and Technology 2(4): 311-317.

Mandal A.K., Sarma P.M., Jeyaseelan C.P., Channashettar V.A., Singh B., Agnihotri A., Lal B. and Datta J. (2014) Large Scale Bioremediation of Petroleum Hydrocarbon Contaminated Waste at Various Installations of ONGC. India: Case Studies. Environmental Research, Engineering and Management 68(2): 41-52. https://doi.org/10.5755/j01.erem.68.2.5632

Moschini L.E., Santos J.E.D. and Pires J.S.R. (2005) Environmental Diagnosis of Risk Areas Related to Gas Stations. Brazilian Archives of Biology and Technology 48(4): 657-666. https:// doi.org/10.1590/S1516-89132005000500019

Nakova R., Baeva G. and Nikolov P. (2004) Competition between maize and Xanthium strumarium L. Pesticidi i Fitomedicina 19(4): 257-263.

Oehlmann J. and Schulte-Oehlmann U. (2003) Molluscs as bioindicators. Chapter 17 of Volume 6: Bioindicators \& Biomonitors: Principles, Concepts and Applications in Markert. Trace Metals and Other Contaminants in the Environment. https:// doi.org/10.1016/S0927-5215(03)80147-9

Parvizi Mosaed H., Sobhanardakani S., Merrikhpour H., Farmany A., Cheraghi M. and Ashorlo S. (2015) The Effect of Urban Fuel Stations on Soil Contamination with Petroleum Hydrocarbons. Iranian Journal of Toxicology 9(30): 1378-1384.

Pearson G. and Oudijk G. (1993) Investigation and Remediation of Petroleum Product Releases from Residential Storage Tanks. Groundwater Monitoring \& Remediation 13(3): 124-128. https://doi.org/10.1111/j.1745-6592.1993.tb00081.x

Pielou E.C. (1966) The Measurement of Diversity in Different Types of Biological Collections. Journal of Theoretical Biology 13: 131-144. https://doi.org/10.1016/0022-5193(66)90013-0

Pinedo J., Ibáñez R., Lijzen JPA., Irabien Á. (2013) Assessment of soil pollution based on total petroleum hydrocarbons and individual oil substances. Journal of Environmental Management 130: 72-79. https://doi.org/10.1016/j.jenvman.2013.08.048

Rhbal H., Souabi S., Safi M., Arad M. and Anouzla A. (2010) Hydrocarbons Diagnostic of Polluted Soils. Scientific Study \& 
Research Chemistry \& Chemical Engineering, Biotechnology, Food Industry 11(4): 449 - 458.

Rusin M. and Gospodarek J. (2016) The Occurrence of Springtails (Collembola) and Spiders (Araneae) as anEffectiveness Indicator of Bioremediation of Soil Contaminated by Petroleum-Derived Substances. International Journal of Environmental Research 10(3): 449-458. https://doi.org/10.22059/ ijer.2016.58764

Saric-Krsmanovic M., Bozic D., Pavlovic D., Elezović I, Vrbnicanin S. (2012) Temperature effects on common cocklebur (Xanthium strumarium L.) seed germination. Romanian Agricultural Research :389-393.

Shannon C.E. and Weaver W. (1964) The Mathematical Theory of Communication. The university of Illinois press, Urbana. $125 \mathrm{p}$.

Sharma G.P., Muhl S.A., Esler K.J. and Milton S.J. (2010) Competitive interactions between the alien invasive annual grass Avena fatua and indigenous herbaceous plants in South African Renosterveld: the role of nitrogen enrichment. Biological Invasions 12(9): 3371-3378. https://doi.org/10.1007/s10530010-9730-y

Solano-Serena F., Marchal R. and Vandecasteele J.P. (2001) Biodégradabilité de l'essence dans l'environnement: de l'évaluation globale au cas des hydrocarbures récalcitrants (Biodegrada- tion of Gasoline in the Environment: From Overall Assessment to the Case of Recalcitrant Hydrocarbons). Oil \& Gas Science and Technology 56(5): 479-498. https://doi.org/10.2516/ ogst:2001039

Sorensen T. (1948) A Method of Establishing Groups of Equal Amplitude in Plant Sociology Based on Similarity of Species and its Application to Analyses of the Vegetation on Danish Commons. Biologiske Skrifter 5(4): 1-34. https://doi.org/10.1016/ S1001-0742(10)60517-7

Van Straalen N. (2004) The Use of Soil Invertebrates in Ecological Surveys of Contaminated Soils. Chapter 6 of Volume 29: Vital Soil: Function, Value and Properties. Developments in Soil Science. https://doi.org/10.1016/S0166-2481(04)80010-2

Tang J., Wang M., Wang F., Sun Q. and Zhou Q. (2011) Eco-toxicity of petroleum hydrocarbon contaminated soil. Journal of Environmental Sciences (China) 23(5): 845-851. https://doi. org/10.1016/S1001-0742(10)60517-7

Vetter J. (2004) Poison hemlock (Conium maculatum L.). Food and Chemical Toxicology 42(9): 1373-1382. https://doi. org/10.1016/j.fct.2004.04.009

Weaver S.E. and Lechowicz M.J. (1983) The Biology of Canadian Weeds. 56. Xanthium strumarium L. Canadian Journal of Plant Science 63(1): 211-225. https://doi.org/10.4141/cjps83-021 